\title{
Bilateral maxillary fused second and third molars: a rare occurrence
}

\author{
Rui-Zhen Liang ${ }^{1,2}$, Jin-Tao $\mathrm{Wu}^{2}$, You-Nong Wu ${ }^{1,2}$, Roger J Smales ${ }^{3}$, Ming $\mathrm{Hu}^{2}$, Jin-Hua Yu ${ }^{1,2}$ \\ and Guang-Dong Zhang ${ }^{1,2}$
}

This case report describes the diagnosis and endodontic therapy of maxillary fused second and third molars, using cone-beam computed tomography (СBCT). A 31-year-old Chinese male, with no contributory medical or family/social history, presented with throbbing pain in the maxillary right molar area following an unsuccessful attempted tooth extraction. Clinical examination revealed what appeared initially to be a damaged large extra cusp on the buccal aspect of the distobuccal cusp of the second molar. However, CBCT revealed that a third molar was fused to the second molar. Unexpectedly, the maxillary left third molar also was fused to the second molar, and the crown of an unerupted supernumerary fourth molar was possibly also fused to the apical root region of the second molar. Operative procedures should not be attempted without adequate radiographic investigation. CBCT allowed the precise location of the root canals of the right maxillary fused molar teeth to permit successful endodontic therapy, confirmed after 6 months. International Journal of Oral Science (2012) 4, 231-234; doi:10.1038/ijos.2012.62; published online 7 December 2012

Keywords: cone-beam computed tomography scans; endodontic therapy; fusion of teeth; maxillary molar

\section{INTRODUCTION}

Fusion is an abnormality of tooth development, defined as the union at the enamel, dentine, cementum or pulp level during different stages of odontogenesis of two or more separately developing contiguous tooth germs. ${ }^{1-3}$ When fusion takes place, the pulp chambers and root canals may either be joined or separated, depending on the stage of tooth development at the time of union. ${ }^{4}$ The process of tooth fusion involves epithelial and mesenchymal germ layers, usually resulting in irregular tooth morphology. ${ }^{5}$ So far as we are aware, this case report is the first to describe rare bilateral fusions observed between maxillary second and third molars, and which also included an unerupted supernumerary fourth molar possibly attached to the apical root region of the left maxillary second molar.

\section{CASE REPORT}

On presentation, a 31-year-old Chinese male complained of a spontaneous, intermittent throbbing pain that had been present for about 1 month in the maxillary right molar area. His medical history was noncontributory. However, following the taking of a panoramic radiograph, he had undergone the failed extraction of a third molar 1 week earlier in a private dental clinic. The dental pulp of the third molar had been exposed during the failed extraction, and pain had continued subsequent to the placement of a restoration.

Clinical examination revealed what appeared initially to be a damaged large extra cusp on the buccal aspect of the distobuccal cusp of the maxillary right second molar, but distinct developmental grooves separated them. Carious lesions were present on the occlusal and distoproximal surfaces of the second molar, and both molars elicited sensitivity responses to cold and electric pulp testing. The non-mobile second molar was slightly tender to percussion, periodontal probing depth was less than $3.0 \mathrm{~mm}$ and there were no swellings present. The patient stated that no other family member had a similar dental condition.

Because of the failed extraction and the insufficient information obtained with the panoramic radiograph taken previously in the private dental clinic, and because of the suspicion of tooth fusion, conebeam computed tomography (CBCT) was thought to be necessary. Therefore, with the informed consent of the patient, to determine the root canal systems of the apparently fused right maxillary second and third molars in a three-dimensional manner, dental imaging using CBCT was undertaken (New Tom VG10048S; Quantitative Radiology s.r.l, Verona, Italy). Though bilateral impacted mandibular third molars and what appeared to be a maxillary left unerupted fourth molar were observed on a reconstructed panoramic radiograph, there were obviously insufficient anatomical details shown in both maxillary second molar regions (Figure 1a). However, axial, sagittal and coronal sections of $0.25 \mathrm{~mm}$ thickness confirmed that the maxillary right second and third molars were fused (Figure 1b-1d) and, unexpectedly, the maxillary left second and third molars, and possibly the unerupted supernumerary fourth molar, also were found to be fused (Figure 1b, 1c and 1e). Three-dimensional reconstructions showed the external morphology of the maxillary right (Figure 1f) and left

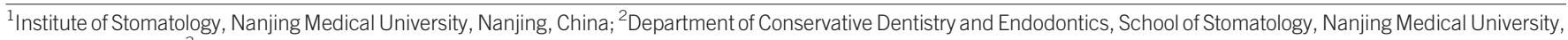
Nanjing, China and ${ }^{3}$ Visiting Research Fellow, Dental School, Faculty of Health Sciences, The University of Adelaide, Adelaide, Australia

Correspondence: Dr GD Zhang, Department of Conservative Dentistry and Endodontics, School of Stomatology, Nanjing Medical University, 136 Hanzhong Road, Nanjing

210029, China

E-mail: egd_zhang@yahoo.com.cn

Received 19 June 2012; accepted 4 November 2012 

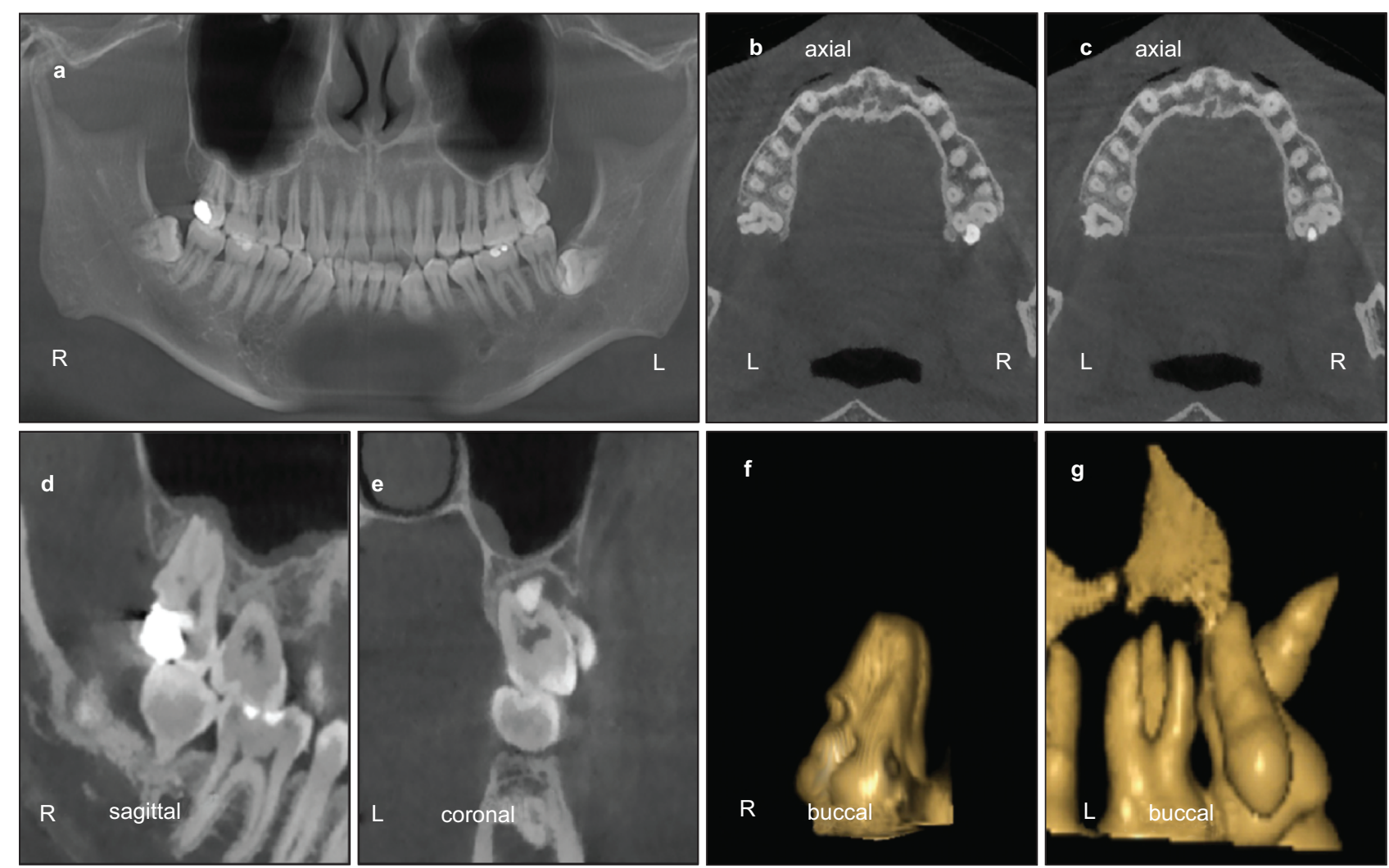

Figure 1 Radiographs and three-dimensional reconstructions. (a) Reconstructed panoramic radiograph. (b and $\mathbf{c}$ ) CBCT axial cross-sections of bilateral maxillary fused second and third molars. (d) CBCT sagittal cross-section of maxillary right fused second and third molars. (e) CBCT coronal cross-section of maxillary left fused second and third molars, and an apically positioned supernumerary tooth. (f) Buccal view of three-dimensional reconstruction of the maxillary right fused teeth. (g) Buccal view of threedimensional reconstruction of the maxillary left fused teeth. CBCT, cone-beam computed tomography.

(Figure 1g) second molar regions viewed from the buccal tooth surfaces. Examination of the CBCT images showed three slightly curved and patent root canals present in the maxillary second molar and a similar single canal in the fused third molar that merged with the distobuccal canal of the second molar.

A diagnosis of chronic pulpitis of the maxillary fused second and third molars was made. After infiltration of $1.5 \mathrm{~mL}$ of $4 \%$ articaine hydrochloride (Primacaine; ACTEON Pierre Rolland, Merignac, France) local anaesthetic, the carious lesions were excavated and two separate occlusal access openings were made (Endo Access Bur; Dentsply Maillefer, Ballaigues, Switzerland) in the second and third molars (Figure 2a). The access openings were then joined by removing the dentine wall between the two pulp chambers. Rubber dam isolation was unsuccessful because of the substantial loss and aberrant anatomy of the coronal tooth structure. Instead, tooth isolation was achieved using cotton rolls and gauze packs to protect the airway, accompanied by judicious suctioning during irrigation of the root canals. Coronal flaring was carried out carefully with sizes 1-4 Gates Glidden drills (MANI Inc., Tochigi, Japan), and the root canal working length determined using an electronic apex locator (Root ZX; J. Morita Mfg Corp., Kyota, Japan), the length being confirmed with a periapical radiograph (Figure 2b). Working lengths of the mesiobuccal, distobuccal and palatal canals of the maxillary second molar were 16.5, 17.0 and $18.0 \mathrm{~mm}$, respectively. The maxillary third molar single root canal was enlarged using a tethered no. 15 C-Pilot file (CC+; VDW $\mathrm{GmbH}$, Munchen, Germany). The single curved canal joined the distobuccal root canal of the second molar at a length of $13 \mathrm{~mm}$. The four canals found in the two molars were cleaned and shaped with Protaper (Dentsply Maillefer, Ballaigues, Switzerland) rotary instruments under irrigation with $2.5 \%$ sodium hypochlorite and $17 \%$ ethylenediaminetetraacetic acid. Canal finishing was performed until a Protaper F2 instrument reached the full working length. After final preparation, the root canals were irrigated again to remove the smear layer, and dried with sterile paper points (Dentsply Maillefer). Calcium hydroxide paste (Calcicur; VOCO GmbH, Cuxhaven, Germany) was placed as a root canal medicament, cotton balls were placed in the canal orifices, and the canal access opening were then sealed with Caviton (GC Corp., Tokyo, Japan). After 1 week, clinical examination confirmed that the fused teeth were completely asymptomatic. The calcium hydroxide paste was removed using ultrasonic irrigation (P5 Newtron; ACTEON Satelec, Merignac, France), with distilled water delivered via a 30-G open-ended nickel titanium needle tip attached to an ultrasonic handpiece (Piezo Pilot, Vista Dental Products) set at a midamplitude power setting of 6 in keeping with the manufacturer's instructions and the root canals obturated by cold lateral compaction of gutta-percha cones (Dentsply (Tianjin) International Trading Co. Ltd, Tianjin, China), using AH Plus root canal sealant (Dentsply DeTrey GmbH, Konstanz, Germany). The access cavity was restored temporarily with Caviton, and a final postoperative periapical radiograph taken (Figure 2c). Two weeks later a fibre post (Coltene/Whaledent Inc., Ohio, USA) was placed, and an all-ceramic crown then constructed as a permanent restoration. Recall appointments were scheduled for 3 and 6 months postoperatively. A periapical radiograph taken after six months showed satisfactory results (Figure $2 \mathrm{~d}$ ). 

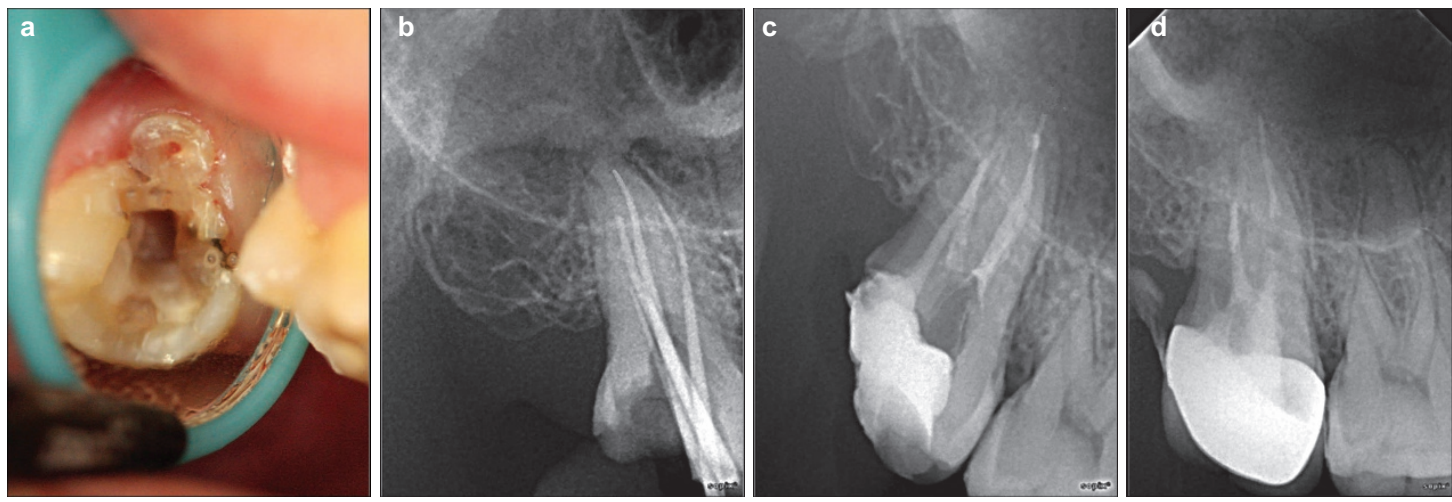

Figure 2 Endodontic therapy in the maxillary right second and third molars. (a) Occlusal view of the initial endodontic access following the removal of caries. (b) Working length determination radiograph. (c) Immediate postoperative radiograph. (d) Postoperative recall radiograph after 6 months.

\section{DISCUSSION}

The prevalence of tooth fusion and germination is estimated to be approximately $0.5 \%-2.5 \%$ in the primary dentition ${ }^{6}$ and even lower in the permanent dentition. ${ }^{7}$ Fusions of teeth may occur in any region of the dental arches, though they more frequently involve anterior teeth and are usually unilateral. ${ }^{6,8}$ Concrescence is a rare form of fusion in which the union to varying degrees of two fully formed adjacent teeth is in cementum only, and is most often observed in maxillary molars. A maxillary third molar and a supernumerary tooth are the teeth most frequently involved. When the cementum union is slight between the affected teeth, they may separate during the extraction of one of the teeth, and the concrescence present may never be noticed. ${ }^{3}$ Fusions of molar teeth, or involving supernumerary teeth, are particularly rare and may result in caries, periodontal disease and crowding. ${ }^{5,9}$

Endodontic treatment is usually problematic with fused molars because of complex root canal anatomy. And, as was apparent in the present case report, the aberrant external tooth morphology and malpositioning may cause difficulties with attempted rubber dam isolation. ${ }^{10}$ Although saliva contamination of root canals introduces microorganisms, there appears to be no evidence from randomised clinical trials that the use of rubber dam isolation is more effective than other isolation methods for the long-term clinical success of root canal therapy. Based on three quoted disparate studies, a recent review of rubber dam usage for endodontic treatment concluded that the 'a negative impact of nonuse of rubber dam on root canal treatment can be indirectly inferred'. ${ }^{11}$ However, the only randomised clinical trial study quoted that involved initial treatments found comparable histologic results for both rubber dam and cotton roll isolation when calcium hydroxide was used for direct pulp capping. ${ }^{12}$ Therefore, apart from effective tooth isolation, effective canal disinfectants and medicaments and temporary restoration seals also appear to be significant factors for reducing microbial contamination of canals during endodontic therapy. ${ }^{13}$

Conventional intraoral periapical radiographs produce only twodimensional images, resulting in superimpositions, incorrect angulations and magnifications of three-dimensional structures. ${ }^{14}$ Therefore, despite having taken a conventional panoramic radiograph, the private practitioner who first treated the patient failed to observe fusion between the maxillary right second and third molars, resulting in an unsuccessful attempted extraction of the third molar. The CBCT images clearly demonstrated the presence of fusion of the maxillary molars and the numbers, positions and morphologies of all root canals associated with each tooth. As a newer diagnostic tool, CBCT can evaluate a tooth in three dimensions and, in selected instances, the additional information obtained justifies the higher irradiation involved compared with fast-speed single conventional intraoral periapical $(1.5-7.0 \mu \mathrm{Sv})$ and extraoral panoramic $(2.7-24.3 \mu \mathrm{Sv})$ radiographs. ${ }^{15-17}$ For dental CBCT units, the effective radiation doses for dento-alveolar and craniofacial fields of view range from 11-674 (median 61) $\mu \mathrm{Sv}$ and from 30-1073 (median 87) $\mu \mathrm{Sv}$, respectively. ${ }^{17}$ As with the present endodontic case report situation, CBCT images were able to provide a better understanding of root canal anatomy, enabling the clinician to identify, clean, shape, and obturate the canals more efficiently. ${ }^{16,18}$ From other relevant reports of using CBCT imaging, nonsurgical endodontic retreatment of a supernumerary tooth that was fused with a maxillary first molar was also successfully performed, ${ }^{19}$ and the successful diagnosis and surgical treatment planning for a supernumerary tooth fused with an impacted mandibular third molar were achieved. ${ }^{20}$

The patient declined surgical extraction of the impacted mandibular molars but, together with the asymptomatic left maxillary fused second and third molars and the unerupted and possibly fused supernumerary fourth molar, they will be reviewed periodically.

\section{CONCLUSIONS}

The present clinical case is a rare example of bilateral fusions of maxillary second and third molars. Clinically, fused teeth may be difficult to detect as, if the enamel is not involved, the visible crowns of the affected teeth may appear normal. Aberrant anatomic tooth structures also may prevent conventional radiography from providing sufficient information for diagnosis and operative treatments. In the present clinical report, three-dimensional reconstructions from CBCT provided invaluable information on the anatomic relations between the right maxillary fused second and third molars, to allow successful endodontic therapy.

\section{ACKNOWLEDGEMENTS}

We appreciate the financial support received from A Project Funded by the Priority Academic Program Development of Jiangsu Higher Education Institutions (2011-137).

1 Velasco LF, de Araujo FB, Ferreira ES et al. Esthetic and functional treatment of a fused permanent tooth: a case report. Quintessence Int 1997; 28(10): 677-680.

2 Garattini G, Crozzoli P, Brenna F. Bilateral dental fusion of the upper central incisors: a multidisciplinary approach. J Esthetic Dent 1999; 11(3): 149-154. 
3 Romito LM. Concrescence: report of a rare case. Oral Surg Oral Med Oral Path Oral Radiol Endod 2004; 97(3): 325-327.

4 Brook AH, Winter GB. Double teeth: a retrospective study of 'geminated' and 'fused' teeth in children. Br Dent J 1970; 129(3): 123-130.

5 Ghoddusi J, Zarei M, Jafarzadeh H. Endodontic treatment of a supernumerary tooth fused to a mandibular second molar: a case report. J Oral Sci 2006; 48(1): 39-41.

6 Blaney TD, Hartwell GR, Bellizzi R. Endodontic management of a fused tooth: a case report. J Endod 1982; 8(5): 227-230.

7 Hülsmann M, Bahr R, Grohmann U. Hemisection and vital treatment of a fused tooth-literature review and case report. Endod Dent Traumatol 1997; 13(6): 253258.

8 Atwan SM, Turner D, Khalid A. Early intervention to remove mesiodens and avoid orthodontic therapy. Gen Dent 2000; 48(2): 166-169.

9 Indra $\mathrm{R}$, Srinivasan MR, Farzana $\mathrm{H}$ et al. Endodontic management of a fused maxillary lateral incisor with a supernumerary tooth: a case report. J Endod 2006; 32(12): 1217-1219.

10 Shafer WG, Hine MK, Levy BM. Developmental disturbances of oral and paraoral structures. In: Shafer WG, Hine MK, Levy BM (eds). A textbook of oral pathology. 4th ed. Philadelphia: Saunders, 1993: 38-39.

11 Ahmad IA. Rubber dam usage for endodontic treatment: a review. Int Endod J 2009; 42(11): 963-972.

12 de Lourdes Rodriques Accorinte M, Reis A, Dourado Longuercio A et al. Influence of rubber dam isolation on human pulp responses after capping with calcium hydroxide and an adhesive system. Quintessence Int 2006; 37(3): 205-212.

13 Abbott PV. Factors associated with continuing pain in endodontics. Aust Dent J 1994; 39(3): 157-161.
14 Silva MA, Wolf $U$, Heinicke $F$ et al. Cone-beam computed tomography for routine orthodontic treatment planning: a radiation dose evaluation. Am J Orthod Dentofac Orthoped 2008; 133(5): 640-645.

15 Scarfe WC, Farman AG, Sukovic P. Clinical applications of cone-beam computed tomography in dental practice. J Can Dent Assoc 2006; 72(1): 75-78.

16 Scarfe WC, Levin MD, Gane D et al. Use of cone beam computed tomography in endodontics. Int J Dent 2009; 2009: 634567.

17 The Seventh Framework Programme of the European Atomic Energy Community (Euratom). Guidelines on CBCT for dental and maxillofacial radiology. Manchester: SEDENTEXCT project, 2012, Available at http://www.sedentexct.eu/content/ guidelines-cbct-dental-and-maxillofacial-radiology (accessed 15 May 2012).

18 La SH, Jung DH, Kim EC et al. Identification of independent middle mesial canal in mandibular first molar using cone-beam computed tomography imaging. J Endod 2010; 36(3): 542-545.

19 Song CK, Chang HS, Min KS. Endodontic management of supernumerary tooth fused with maxillary first molar by using cone-beam computed tomography. J Endod 2010; 36(11): 1901-1904.

20 Ferreira-Junior O, de Avila LD, Sampieri MB et al. Impacted lower third molar fused with a supernumerary tooth-diagnosis and treatment planning using cone-beam computed tomography. Int J Oral Sci 2009; 1(4): 224-228.

cc This work is licensed under a Creative Commons SOMERIIHISHESERVED Attribution-NonCommercial-NoDerivative Works 3.0 Unported License. To view a copy of this license, visit http:// creativecommons.org/licenses/by-nc-nd/3.0 